\title{
Structures of the Covariance Matrices in the Classifier Design
}

\author{
Šarūnas Raudys and Aušra Saudargienè \\ Institute of Mathematics and Informatics \\ Akademijos 4, Vilnius 2600, Lithuania \\ e-mail: raudys@klt.mii.lt
}

\begin{abstract}
Structurization of the covariance matrices helps to rednce a number of parameters to be estimated. When assumptions on the structure of the matrix are correct the structurization of the covariance matrix helps to reduce the generalization error in small learning-set cases. Efficacy of the matrix structurization increases if one decorrelates and scales the data, and uses the optimally stopped single layer perceptron classifier afterwards.
\end{abstract}

Index terms: regularized discriminamt analysis, learning-set size, generalization, dimensionality, covariance matrix, parameters reduction, single layer perceptron.

\section{Introduction.}

An essential factor while designing any pattern recognition system is a lcarning-set size / dimensionality ratio. In a standard linear and quadratic discrimianant analysis, one needs to estimate populations covariance matrices and invert them. When $p$, the dimensionality of the feature vector, exceeds $n$, the number of observations used to estimate the covariance matrix $\mathbf{S}$, this matrix becomes singular and one can not invert it. Similar problem arise when $n$ is close to $p$.

There is a number of ways to overcome this kind of difficulties (sce e.g. Raudys, 1991). We'll catcgorise these tcchniques into following three groups:

a) dimensionality reduction by feature extraction or feature selection,

b) regularization of the sample covariance matrix. The simplest and the most popular example is a use of the shrinkage (ridge) cstimate

$$
\mathbf{S}^{\mathrm{RDA}}=\mathrm{S}+\lambda \mathbf{I} \text {, }
$$

where $\mathbf{S}$ is the conventional maximum likelihood estimate of the covariance matrix $\Sigma, I$ is a $p \times p$ identity matrix and $\lambda$ is a positive regularization constant (Fricdman, 1989, McLachlan, 1992),

c) structurization of a truc covariance matrix $\Sigma$, and its description by a small number of parameters. Examples are assumption that $\Sigma$ is a diagonal matrix, it has a block structure, $\Sigma$ is a Toeplitz matrix of general form, $\Sigma$ is circular, $\Sigma$ describes an autoregression process, e.t.c.

From theoretical studies it is known small sample properties of the statistical pattern classifiers depend on a number of parameters $r$ used to characterise the covariance matrix. When the number of parameters $r$ is proportional to dimensionality $p$, asymptotically for large $n, r$ and $p$. estimation of the common for both pattern classes covariance matrix (CM) $\Sigma$ does not affect the increase in the 
generalization error (Raudys, 1972, Deev, 1974, Meshalkin \& Serdobolskij, 1978, see also Raudys \& Pikelis, 1980; Raudys \& Jain, 1991). It means, for large $n, r$ and $p$, one can expect high efficacy of structurization of the covariance matrix .

In this paper we'll analyse the third group of techniques more thoroughly. We consider several popular and unpopular stnuctures of CM discussed in Raudys (1991) and show that when assumptions on the structure of the matrix are correct the sinucturization of CM helps to reduce the gencralization crror in small lcarning-sct cases. Efficacy of the matrix structurization increases if onc uses the information about the structure in order to decorrelate and normalise the data, and uses the optimally stopped single layer perceptron classifier afterwards.

\section{Notations and abbreviations:}

$N$ - number of learning vectors in one class, $p$ - number of the features (dimensionality),

RDA - regularized discriminant analysis, LDA - linear discriminant analysis, EDC - Euclidean dislance classifier, AR - auloregression modcl, OFS - original feature space, TFS - transformed feature spacc, SLP - single layer perceptron, $\mathrm{CM}$ - covariance matrix,

\section{Structures of the covariance matrix.}

Use of the Gaussian model to describe the distribution density of the pattern classes leads to the quadratic discriminant function. In two category case, one needs to estimate two $p$-variate mean vectors and two $p \times p$ covariance matrices, altogether $2 p+p(p+1)$ parameters. An assumption that the pattern classes share the common $\mathrm{CM}$ $\Sigma$ lcads to the standard Fisher lincar discriminant function (DF) with smaller number of parameters to be cstimated from the learning-set

$$
\left.g(x)=\left(x-\frac{1}{2}\left(\bar{x}^{(1)}+\bar{x}^{(2)}\right)\right)^{\prime} S^{-1}\left(\bar{x}^{(1)}-\bar{x}^{(2)}\right)\right),
$$

where

$\overline{\mathbf{x}}^{(1)}, \overline{\mathbf{x}}^{(2)}$ are sample means,

$\mathbf{S}$ is the sample estimate of the covariance matrix, and $\mathbf{x}=\left(x_{1}, \ldots, x_{p}\right)^{\prime}$ is a $p$-variate vector to be classified.

When one assumes that the features are independent, instead of $S$ one uses a diagonal variance matrix $D$ composed from diagonal elcments of $S$. In certain applications, the features can be grouped into blocks of the features, and one of possible ways to reduce the number of parameters is to assume that the blocks are statistically independent. In the high dimensional cases, the number of parameters required to characterise $\mathrm{CM}$ 


$$
\Sigma^{\mathrm{BL}}=\left[\begin{array}{cccc}
\Sigma_{1} & 0 & \cdots & 0 \\
0 & \Sigma_{2} & \cdots & 0 \\
\cdots & \cdots & \cdots & \cdots \\
0 & 0 & \cdots & \Sigma_{h}
\end{array}\right]
$$

is reduced immensely.

An interesting and useful model which requires a small number of parameters to describe a joint dependence between all $p$ variables is a model based on an approximation of a joint probability distribution by the first order tree dependence

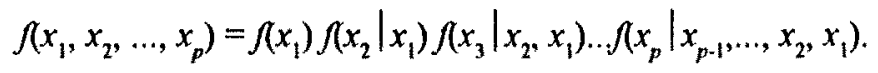

In this model, it is assumed that cach variable is conditioned upon, at most. one another variable. Then probability density function (4) can be written in the following form:

$$
f\left(x_{1}, x_{2}, \ldots, x_{p}\right)=\prod_{j=1}^{p} f\left(x_{j} \mid x_{m_{j}}\right) \quad\left(0 \leq m_{j} \leq p\right)
$$

where a sequence $m_{2}, \ldots, m_{p}$ constitutes a graph of connections (an unknown permutation of the integers $1,2, \ldots, p)$ and $f\left(x_{i} \mid x_{0}\right)$, by definition is equal to $f\left(x_{i}\right)$. In a general case, the covariance matrix have $p * p$ non-zcro clements. An inverse of this matrix $\Sigma^{-1}$ which has to be used to design the classificr, however, has only $2 p$ different non-zero elements. It is a result of the assumption that each component of the vector $x$ depends only on one another component. This important model lacked to be described in all pattern recognition textbooks and remained practically unnoticed in pattern recognition community.

There is a number of models that allow to evaluate temporal or spatial dependence between the data points. The recognition of the temporal and spatial objects and phenomena requires to work with vectors of very high dimensionality. It is an advantageous area of application of the constrained statistical classifiers. Therefore an expertise gained in this knowledge area probably can be useful in ANN design.

Let the components $x_{1}, x_{2}, \ldots, x_{p-1}, x_{p}$ of the multivariate vector $\mathbf{x}$ are measurements differing in time or in space, and assume they are stationary random process. Then the covariance matrix has Tocplitz struclurc. Only $p$ parameters $\left(\delta_{1}\right.$, $\delta_{2}, \ldots, \delta_{p}$ ) describe the structure of dependence between the variables. A number of special models, such as circular, autoregression, moving average, ARMA, and others, allow to reduce the number of parameters even more. In the circular model. we have a symmetry: $\delta_{2}=\delta_{p}, \delta_{3}=\delta_{p-1}, \delta_{1}=\delta_{p-2}$, e.t.c. This model is competent to estimate random periodical processes. $A$ number of parameters $q$ in the autorgression model, depends of the model's order, and typically $q<<p$. 


\section{Simulation experiments.}

The efficacy of structurization of the covariance matrix was performed by means of simulation. In the experiments, we estimated parameters of different modifications of the linear discriminant function by using different randomly chosen Icarning-sets and estimated the gencralization error on a large test-set (for a real world data) or calculated it analytically (for an artificial Gaussian data). Two category case was analysed.

Following linear classifiers were analysed in this section:

- RDA - the standard linear regularized discriminant analysis (RDA) with the

optimal $\lambda$ evaluated from 50 cstimates of the generalization error,

- the standard linear Fisher classifier (when $n<p$. we used a pscudoinverse

of the covariance matrix),

- EDC - the Euclidean distance classifier, where it is assumed $\Sigma=\sigma^{2} \mathbf{I}$,

- Tree - LDA with the trec type dependence structured covariance matrix,

- Tocp - LDA with the Tocplity structured covariance matrix.

- Circ - LDA with the circular structurcd covariance matrix,

- ARI - LDA with the first order autoregression structured covariance matrix,

- AR4 - LDA with the fourth order autoregression structured covariance matrix.

We concentrated our analysis mainly on a case when the number of learning examples $n=N_{1}+N_{2}=2 N$ is smaller than the number of dimensions $p$ of the feature vector. Therefore in our investigation, we used 40 -variate artificial Gaussian data similar to two 40-variate Friedman (1989) Gaussian data sets used in analysis of the linear RDA where the first or last features were most informative. In our analysis we used also cleven 40-variate Gaussian data sets where various types of assumptions on the structure of the covariance matrices were fulfilled.

In addition to 13 artificial data types, in our research, we also used five real world data sets. In 28-variatc (spectral and cepstral features) vowels data, we had 400 vowels in one class pronounced by 20 speakers, and in 66-variate (spectral and cepstral features) lung noise data, we had 180 vectors measured on 18 paticnts in onc class. 65-variate (shape, size, histogram statistics, Gabor wavelet response, elc.) mammogram data set consists of 57 bening and 29 malignant mammograms, and 60variate (cnergy within a particular frequency over a certain period of time) sonar data set represents two classes that describe sonar signals bounced off a metal cylinder and those bounced off a cylindrical rock (111 and 97 pattcrus, respectively). Two class 33-variate ionosphere data set contains 127 and 226 patterns. While training the classificrs, we have chosen $N$ vectors from each class randomly, and tested the classifiers on all vectors. Asymptotic error rates obtained by using different structurization methods are summarized in Table 1.

In all experiments with artificial Gaussian data we have chosen $N_{1}=N_{2}=$ $N=13$, the same dimensionality/ sample size ratio as in the Fricdman's experiments with the LDA. All experiments were performed 25 times with each size of the learning-sct. 
Table 1. Asymptolic error rates of the data.

\begin{tabular}{|c|c|c|c|c|c|c|c|c|}
\hline No & Model & Duta & LDA & Circ & Toepl & Tree & ARI & AR4 \\
\hline 1 & Circ. 1 & $\mathrm{C} 30$ & 0,03 & 0,03 & 0,03 & 0,05 & 0,28 & 0,21 \\
\hline 2 & Circ. 2 & C15 & 0,03 & 0,03 & 0,03 & 0,05 & 0,19 & 0,14 \\
\hline 3 & Circ. 3 & $\mathrm{C} 05$ & 0,03 & 0,03 & 0,03 & 0,04 & 0,06 & 0,05 \\
\hline 4 & Toepl 1 & T1105 & 0,03 & 0,05 & 0,03 & 0,04 & 0,06 & 0,05 \\
\hline 5 & Toepl 2 & $\mathrm{~T} 1054$ & 0,03 & 0,05 & 0,03 & 0,04 & 0,04 & 0,04 \\
\hline 6 & Toepl 3 & T31010 & 0,03 & 0,04 & 0,03 & 0,03 & 0,03 & 0,03 \\
\hline 7 & Tree 1 & Treel & 0,03 & 0,09 & 0,09 & 0,03 & 0,06 & 0,09 \\
\hline 8 & Tree 2 & Tree2 & 0,03 & 0,28 & 0,29 & 0,03 & 0,15 & 0,28 \\
\hline 9 & Tree 3 & Tree3 & 0,03 & 0,10 & 0,10 & 0,03 & 0,07 & 0,10 \\
\hline 10 & $\Lambda \mathrm{R} 1$ & AR046 & 0,03 & 0,03 & 0,03 & 0,03 & 0,03 & 0,03 \\
\hline 11 & AR 4 & AR034 & 0,03 & 0,04 & 0,03 & 0,03 & 0,07 & 0,03 \\
\hline 12 & Fried $\mathrm{f}$ & F $\mathrm{MA}$ & 0,03 & 0,10 & 0,10 & 0,09 & 0,30 & 0,10 \\
\hline 13 & Fried! & FaMI & 0,03 & 0,04 & 0,04 & 0,04 & 0,30 & 0,04 \\
\hline 14 & Wowel & Wovels & 0,01 & 0,06 & 0,05 & 0,05 & 0,06 & 0,07 \\
\hline 15 & Lung & Lung & 0,05 & 0,23 & 0,23 & 0,26 & 0,29 & 0,30 \\
\hline 16 & Mamm. & Mamm. & 0.01 & 0.01 & 0.01 & 0.01 & 0.01 & 0.01 \\
\hline 17 & Sonar & Sonar & 0.087 & 0.164 & 0.149 & 0.221 & 0.188 & 0.173 \\
\hline 18 & Ionosph & Ionosph & 0.103 & 0.145 & 0108 & 0.137 & 0.177 & 0.145 \\
\hline
\end{tabular}

Results are presented in Table 2 . In the first column, we present the code of the data and $N$, the learning set size (for the real world data). In the second one, we present mean values of the generalization error of the standard RDA with optimal value of $\lambda$. In following columns, we present relative eficacies of different classifiers: the generalization error of our bench-mark method - the standard RDA divided by the generalization error of the classifier under consideration in this column: $\gamma=P_{n}^{R D A} / N_{n}^{\text {clamifici }}$. Gamma $(\gamma)$ values are presented in bold for these Gaussian models where assumptions of the covariance matrices structure are correct.

From the simulation experiments we see the RDA always outperform the standard methods: the Fisher LDA and EDC. When assumptions on the structure of the matrix are correct the structurization of the covariance matrix often helps to reduce the gencralization error in small learning-set cases. In some cases, the gain can be very high. E.g. for data AR( 334 we obtained a gain - 4.59 times on average for 25 randomly chosen learning sets. For some configurations of the data and lcarningset sizes, even for cases when the postulated dependence model was correct, the structurization resulted no gain in comparison with the regularized discriminant analysis. Examples are all Gaussian data sets gencrated according the Tocplitz. model. For these three data models even simple EDC performed better than the linear DF with the structured covariance matrix.

Structurization by the first order tree dependence, the Toeplitc, AR and circular models resulted no gain while applied to all real data sets. For the lung data the representation of $66 \times 66$ covariance matrix in a block form (3) composed from six $11 \times 11$ blocks in the diagonal resulted the asymptotic error 0.13 , and helped to obtain a certain gain: in 25 cxperiments with learning sets of size $N=22$ the 
gencralization error was decreased 1.20 times $\left(E P_{n}{ }^{\mathrm{RDA}}=0.24\right)$, for $N=33-1.31$ times $\left(E P_{n}{ }^{\mathrm{RDA}}=0.23\right)$, however for $N=132, \gamma=0.52\left(E P_{n} \mathrm{RDA}=0.07\right)$.

Table 2. The mean gencralization error $E P_{n}^{\text {RnA }}$ of the standard lincar RDA and the relative efficacies $\gamma=P_{n}^{\text {RDA }} / P_{n}^{\text {clasinf }}$ of the Fisher classifier, EDC, and LDA with use of different structured covariance matrices.

Classif. EPRDA $\quad \gamma$ Euclid $\gamma$ F\&Circ $\quad \gamma$ F\&Tp $\quad \gamma$ F\&Tree $\quad \gamma$ F\&ARI $\quad \gamma$ F\&AR4 $\quad \gamma$ Fisher Method

Data

\begin{tabular}{|c|c|c|c|c|c|c|c|c|}
\hline C30 & 0,12 & 0,43 & 1,95 & 0,55 & 0,69 & 0,43 & 0,43 & 0,63 \\
\hline C15 & 0,11 & 0,62 & 1,76 & 0,75 & 0,8 & 0,62 & 0,62 & 0,70 \\
\hline $\mathrm{C} 05$ & 0,05 & 0,95 & 0,92 & 0,72 & 0,73 & 0,87 & 0,81 & 0,43 \\
\hline T1105 & 0,05 & 0,9 & 0,59 & 0,67 & 0,81 & 0,89 & 0,83 & 0,41 \\
\hline T1054 & 0,05 & 0,96 & 0,6 & 0,69 & 0,67 & 0.83 & 0,45 & 0,38 \\
\hline 131010 & 0,04 & 0,98 & 0,55 & 0,55 & 0,51 & 0,71 & 0,56 & 0,32 \\
\hline 'Treel & 0,15 & 0,72 & 0,91 & 0,88 & 1,1 & 0,76 & 0,92 & 0,58 \\
\hline Tree2 & 0,2 & 0,77 & 0,95 & 0,92 & 1,6 & 0,74 & 1,00 & 0,58 \\
\hline Tree3 & 0,3 & 0,62 & 0,67 & 0,67 & 1,23 & 0,62 & 0,64 & 0,75 \\
\hline AR046 & 0,16 & 0,76 & 2,26 & 2,15 & 1,15 & 2,65 & 2,9 & 0,55 \\
\hline AR034 & 0,26 & 0,60 & 3,08 & 3,70 & 1,02 & 0,63 & 4,59 & 0,62 \\
\hline F $\lambda M C$ & 0,21 & 0,84 & 0,83 & 0,83 & 1,03 & 0,84 & 0,86 & 0,67 \\
\hline$F \lambda M 1$ & 0,05 & 0,99 & 0,97 & 0,91 & 0,67 & 0,99 & 0,99 & 0,31 \\
\hline Wov.9 & 0,08 & 0,70 & 0,90 & 0,99 & 0,91 & 0,74 & 0,62 & 0,49 \\
\hline Wov. 14 & 0,07 & 0,7 & 0,89 & 0,83 & 0,82 & 0,77 & 0,65 & 0,27 \\
\hline Wov.56 & 0,03 & 0,35 & 0,44 & 0,53 & 0,48 & 0,39 & 0,35 & 0,63 \\
\hline $\mathrm{Ma} 10$ & 0,18 & 0,55 & 0,65 & 0,63 & 0,97 & 0,61 & 0,62 & 0,66 \\
\hline $\operatorname{Ma} 20$ & 0,09 & 0,37 & 0,42 & 0,43 & 0,84 & 0,39 & 0,41 & 0,54 \\
\hline Ma 25 & 0,07 & 0,26 & 0,32 & 0,33 & 0,69 & 0,32 & 0,32 & 0,51 \\
\hline Son20 & 0,22 & 0,70 & 0,80 & 0,74 & 0,83 & 0,80 & 0,78 & 0,8 \\
\hline Son 30 & 0,19 & 0,68 & 0,79 & 0,74 & 0,76 & 0,77 & 0,76 & 0,65 \\
\hline Son 80 & 0,11 & 0,41 & 0,66 & 0,65 & 0,55 & 0,60 & 0,58 & 0,92 \\
\hline Ionoll & 0,16 & 0,72 & 0,86 & 0,74 & 0,88 & 0,78 & 0,89 & 0,64 \\
\hline Ionol6 & 0,14 & 0,67 & 0,79 & 0,73 & 0,82 & 0,72 & 0,81 & 0,52 \\
\hline Iono66 & 0,10 & 0,57 & 0,75 & 0,91 & 0,79 & 0,60 & 0,76 & 0,90 \\
\hline
\end{tabular}

\section{Data transformations and the single layer perceptron.}

Recently it became known, that after the first total gradient training itcration of the single layer perceptron (SLP), one can obtain the Euclidean distance classifier and move further towards RDA and the standard Fisher linear DF if certain conditions are satisfied (the centre of the data is moved to the zero point, for $N_{2}=N_{1}$ one uses symmetrical targets for both pattern classes, starts training from zero weights, and uses the total gradient training). Thus, if the training is successful and one succeds to stop training optimally, onc can obtain the optimal RDA by using this iterative numerical method. In further training, the SLP classificr can move towards the minimum empirical crror and the support vector (maximum margin) 
classificrs (Raudys, 1996, 1998). Therefore, if the data differs from Gaussian with common covariance matrix then, in principle, in further training one can expect to obtain smaller gencralization crror.

ltcrative training of the single layer perceptron becomes difficult when variances of the data are different in various directions; it means when eigenvalues of the covariance matrix $\Sigma$ are essentially different. We can try to equalise the variances by transforming the data by means of rotation and scaling: $\mathbf{y}=\mathbf{D}^{-1 / 2} \mathbf{T}$ ' $x$, where $D$ and $\mathrm{T}$ are $p \times p$ diagonal eigenvalue and $p \times p$ eigenvectors matrix of the sample covariance matrix $S$. Then sample covariance matrix $S_{y}$ of the vector $y$ will be the identity matrix. Affer the first learning itcration we obtain the discrimianant function

$\left.\left.g(\mathbf{y})=\left(\mathbf{y}-\frac{1}{2}\left(\overline{\mathbf{y}}^{(1)}+\overline{\mathbf{y}}^{(2)}\right)\right)^{\prime}\left(\overline{\mathbf{y}}^{(1)}-\overline{\mathbf{y}}^{(2)}\right)\right) k_{\mathrm{B}}=\left(\mathbf{x}-\frac{1}{2}\left(\overline{\mathbf{x}}^{(1)}+\overline{\mathbf{x}}^{(2)}\right)\right)^{\prime} \mathbf{S}^{-1}\left(\overline{\mathbf{x}}^{(1)}-\overline{\mathbf{x}}^{(2)}\right)\right) k_{\mathrm{B}}$,

where $\overline{\mathbf{y}}^{(1)}=\mathbf{D}^{-1 / 2} \mathbf{T}^{\prime} \overline{\mathbf{x}}^{(1)}, \overline{\mathbf{y}}^{(2)}=\mathbf{D}^{-1 / 2} \mathbf{T}^{\prime} \overline{\mathbf{x}}^{(2)}$, and $k_{\mathrm{II}}$ is a constant.

It means, after the training in the y space, anter the first itcration we obtain the classifier that is equivalent to the Euclidean distance classifier in the transformed (y) space (TFS), and the standard linear Fisher DF in the original (x) feature space. Let now transform the data by means of the matrix $G_{R D A}=(D+\lambda I)^{-1 / 2} T: y=G_{R D A} X$. Then after the first iteration we obtain the classifier that is equivalent to RDA analysis in the $\mathbf{x}$ space. When we transform the data by means of matrix $\mathbf{G}_{\mathrm{TREE}}=$ $\mathbf{D}_{\text {TRER }}^{-1 / 2} \mathbf{T}_{\text {TRRE }}: \mathbf{y}=\mathbf{G}_{\mathrm{TRER}} \mathbf{x}$, then after the first iteration we obtain the classificr that is cquivalent to the LDA with tree struclured covariance matrix in the original feature space (OFS). In above equation, $\mathbf{D}_{\text {TRrs }}$ and $T_{\text {TRER }}$ are are $p \times p$ diagonal eigenvalue and $p \times p$ eigenvectors matrix of the trec structurized cstimate of the covariance matrix. The same considerations are valid for other structurization methods. When the data is Gaussian with the common for all classes covariance matrix, and the postulated feature dependence model is correct, then there is a small chance to reduce the generalization error in further training. For a non-Gaussian data, models with different $\mathrm{CM}$ in both pattern classes, and cases when one postulates the structure of CM incorrectly, however, an additional use of SLP can result a certain success.

Therefore, in a second part of our experimental work, we tested the nonlincar SLP. In simulation experiments we translated the learning data centre $0.5\left(\overline{\mathbf{x}}^{(1)}+\overline{\mathbf{x}}^{(2)}\right)$ into the zero point, initialised the SLP with the zero weight vector, used the sigmoid activation function and trained the perceptron in a batch-mode with a standard back propagation algorithm using targets 0 and 1 . In experiments with small lcarning sets, in order to obtain a large margin quickly, we increased the learning step $\eta$ progressively with each iteration number $t: \eta=0.2^{*} 1.03^{t} ; t_{\max }=500$. We trained the SLP in the original $(x)$ space and afterwards in the transformed space $(y=T x)$. In each experiment, we determined an optimal stopping moment $t_{\text {opt }}$ from estimates of the generalization error obtained either from the test-set data (for the real world data) or calculated analytically (for Gaussian data). Results are presented in Table 3. 
Table 3. The mean generalization error $E P_{n}{ }^{\mathrm{RDA}}$ of the standard lincar RDA and the relative cficacies $\gamma=P_{n}^{\mathrm{knA}} / P_{n}^{\text {ederifte }}$ of the SLP classifier without and after the data transformation performed by use of differenily structured covariance matrices.

\begin{tabular}{|c|c|c|c|c|c|c|c|c|}
\hline $\begin{array}{l}\text { Class. } \\
\text { Method } \\
\text { Data }\end{array}$ & $E P^{R D A}$ & $\begin{array}{l}\gamma \text { SLP } \\
\text { in OFS }\end{array}$ & $\begin{array}{l}\text { y SLP } \\
\text { in TTS } \\
\text { by } T_{\text {Circ }}\end{array}$ & $\begin{array}{l}\gamma \text { SLP } \\
\text { in TFS } \\
\text { by } \mathbf{T}_{\text {Top }}\end{array}$ & $\begin{array}{l}y \text { SLP } \\
\text { in TTS } \\
\text { by } \mathbf{T}_{\text {Tree }}\end{array}$ & $\begin{array}{l}\gamma \mathrm{SLP} \\
\text { in } \mathrm{TFS} \\
\text { by } \mathrm{T}_{\mathrm{ARI}}\end{array}$ & $\begin{array}{l}\gamma \mathrm{SLP} \\
\text { in TFS } \\
\text { by TAR4 }\end{array}$ & $\begin{array}{l}\gamma \mathrm{SIP} \\
\text { in TTS } \\
\text { by } \mathrm{T}_{\text {conv }}\end{array}$ \\
\hline C30 & 0,12 & 0,9 & 1,97 & 0,78 & 1,08 & 0,5 & 0,49 & 0,38 \\
\hline $\mathrm{C} 15$ & 0,11 & 0,91 & 1,77 & 0,98 & 0,88 & 0,67 & 0,66 & 0,36 \\
\hline $\mathrm{C} 05$ & 0,05 & 0,99 & 0,92 & 0,74 & 0,78 & 0,87 & 0,82 & 0,2 \\
\hline TI105 & 0,05 & 1,00 & 0,64 & 0,71 & 0,89 & 0,94 & 0,85 & 0,19 \\
\hline T1054 & 0,05 & 1,00 & 0,62 & 0,7 & 0,65 & 0,84 & 0,48 & 0,16 \\
\hline T31010 & 0,04 & 1,00 & 0,56 & 0,56 & 0,62 & 0,72 & 0,57 & 0,14 \\
\hline Treel & 0,15 & 1,00 & 1,13 & 1,09 & 1,21 & 1,05 & 1,18 & 0,47 \\
\hline Trce2 & 0,2 & 0,98 & 1,12 & 1,08 & 1,68 & 0,95 & 1,18 & 0,57 \\
\hline Tree? & 0,3 & 0,94 & 1,07 & 0,99 & 1,54 & 0,9 & 1,09 & 0,78 \\
\hline AR046 & 0,16 & 1,00 & 2,29 & 2,17 & 1,22 & 2,67 & 2,92 & 0,45 \\
\hline AR034 & 0,26 & 0,89 & 3,45 & 3,84 & 1,23 & 1,05 & 4,62 & 0,67 \\
\hline $\mathrm{F} \lambda \mathrm{M}\lceil$ & 0,21 & 0,96 & 0,96 & 0,96 & 1,09 & 0,97 & 0,98 & 0,63 \\
\hline $\mathrm{F} \lambda \mathrm{M} 1$ & 0,05 & 1,01 & 0,98 & 0,92 & 0,69 & 1,00 & 1,00 & 0,21 \\
\hline Wov.9 & 0,08 & 0,97 & 1,09 & 1,19 & 0,99 & 0,91 & 0,8 & 0,32 \\
\hline Wov 14 & 0,07 & 1,06 & 1,08 & 1,05 & 0,99 & 0,97 & 0,88 & 0,2 \\
\hline Wov. 56 & 0,03 & 1,05 & 1,12 & 1,1 & 1,08 & 1,03 & 1,05 & 0,84 \\
\hline $\mathrm{Ma} 10$ & 0,18 & 0,98 & 1,02 & 0,96 & 1,2 & 0,96 & 0,98 & 0,71 \\
\hline $\operatorname{Ma} 20$ & 0,09 & 1,02 & 1,11 & 1,02 & 1,64 & 1,07 & 1,04 & 0,47 \\
\hline $\operatorname{Ma} 25$ & 0,07 & 1,19 & 1,44 & 1,33 & 1,41 & 1,54 & 1,38 & 0,43 \\
\hline Son 20 & 0,22 & 1,08 & 0,98 & 0,96 & 0,96 & 0,99 & 0,99 & 0,66 \\
\hline Son 30 & 0,19 & 1,11 & 1,03 & 1,03 & 0,99 & 1,04 & 1,04 & 0,61 \\
\hline Son 80 & 0,11 & 1,45 & 1,98 & 1,93 & 1,93 & 1,93 & 1,94 & 1,89 \\
\hline Ionoll & 0,16 & 1,00 & 1,01 & 0,99 & 0,96 & 1,05 & 1,13 & 0,54 \\
\hline Iono 16 & 0,14 & 1,05 & $1,0 !$ & 0,97 & 0,94 & 1,06 & 1,1 & 0,48 \\
\hline Iono66 & 0,1 & 1,31 & 1,35 & 1,32 & 1,36 & 1,29 & 1,33 & 1,23 \\
\hline
\end{tabular}

In the Table we have the average results. The results advocate, the efficacy of the optimally stopped SLP in OFS is almost the same as that of the RDA. Higher values of the generalization error of the SLP typically were associated with these few cases when 500 itcrations were not sufficient to train the perceptron. It is worth to note that after the transformation $\mathbf{y}=\mathbf{T x}$, we obtained a significant increase in the Icarning specd: typically only few training iterations were sufficient to obtain the smallest gcieralization error.

Practically in all experiments, the transformations of the data according the structured estimate of the sample CM and subsequent use of the optimally stopped SLP helped to improve efficacy of the covariance matrix structurization. E.g. for the Gaussian data $\mathrm{C} 30$ the additional use of the data transformation based circular model and SLP helped to reduce the generalization error 1.97 times in comparison with the best statistical method - RDA. For some models additional use of SLP leads to a significant gain: e.g. with tree type dependence model we had athe gain $\gamma \mathrm{Tree}=1.23$, 
and now $\gamma_{\text {Trookst. }}=1.56$. For Tocplitz model, however, possibly, we have chosen too "difficuli" model and estimated model's parameters incficiently and did not obtaincd any gain: c.g. $\gamma_{\text {F\&lop }}=0.67$, and $\gamma$ SLP in TS by $\mathbf{T}_{\text {Toeplite }}=0.71$, however for other syntetic data (AR034) the Toeplitz model structurization resulted a significant gain: $\gamma$ SLP in TFS by $\mathbf{T}_{\text {Toeplitc }}=3.84$.

Similar observations we have for the real world data too. For the wovels data and learning-set sizes 9, 14 and 56 for the Toeplitz model we have had nLDA\&Toep $=0.99,0.83$, and 0.53 , i.e. no improvement in comparison with RDA. Now, with the data transformations and subsequent SLP training, we have $\gamma$ SLP in TS by $\mathbf{T}_{\text {Tooplite }}=1.19,1.05$, and 1.10. For the lung data and learning-set sizes 22,33 and 132 for the block matrix model we have had $\gamma_{F} \&$ Tocp $=1.20,1.31$ and 0.52 . After the transformations and SLP we have $\gamma$ SLP in TS by $\mathbf{T}_{\text {Teeplite }}=1.36,1.62$, and 2.05 , i.c., an obvious improvememt.

\section{Concluding remarks.}

The efficacy of the structurization of the covariance matrix depends both on the data as well as on peculiarities of the particular learning-set. In a part of the experiments, we obtained no or very insignificant gain.

In our comparative experiments, we used optimal values of $\lambda$ for the standard RDA cstimated from the test-sct, or analytically for the Gaussian pattern classes. Therefore our experiments result optimistic estimates of the efficacy of the regularized discriminant analysis and pessimistic estimates of our efficacy coefficients $\gamma=P_{n}{ }^{\mathrm{RDA}} / P_{n}{ }^{\text {classifier }}$. Nevertheless, we see that for certain Gaussian models use of correct assumptions on the structure covariance matrix result an obvious gain. In most cases, structurization outperform the standard linear discriminant analysis, and in some cases, it loses against the regularized discriminant analysis. Therefore in practical applications, one obligatory must use an additional validation-set in order to decide which classification method to use. In extremely high-dimensional cases, however, one can expect these parameters can be estimated by the leaving-one out or rolation methods. The situation is analogous to RDA where we have to choose the optimal valuc of the regularization parameter $\lambda$.

Our experiments with the artificial and real world data sets have demonstrated that the efficacy of the matrix structurization increases if one uses the information about the structure in order to decorrelate and scalc the data, and uscs the optimally stopped single layer perceptron classifier afterwards. It is a new way to incorporate an additional, the statistical, information in the perceptron training process. We see, that it is useful to structurize the covariance matrix. More statistical models and more real world data sets should be analysed in future research work. Special numerical calculation schemes that speed up the calculations in the model validation slage should be developed.

In present paper we analysed the eflicacy of the structurization of the covariance matrix and the joint use of the data transformation and the SLP in the 
linear discriminant analysis problem only. No doubt, this structurization of the sample covariance matrix can be used in pattern classification with different covariance matrices as well as in regression tasks.

\section{Acknowledgement.}

The authors are thankful to Dr. Algimantas Rudzionis from Kaunas University of Technology, Dr. Bulent Sankur from Istanbul Bogazici University, Prof. Mineichi Kudo from Hokaydo University, and Professor Jack Sklansky from UCA, Irvine for providing the real world data sets for the experiments.

\section{References.}

Deev, A.D. (1974). Discriminant function designed on independent blocks of variables. - Proc. Acad. of Sci. of USSR, Eng. ('ybernetics, (USSR J.), 12, 153-156 (in Russian).

Friedman J.M.(1989). Regularized discriminant analysis. J. American Statistical Association, 84, 165-175.

McLachlan, G.J.(1992). Discriminant Analysis and Statistical Pattern Recognition. Willey.

Mcshalkin, L.D., \& Scrdobolskij, V.I. (1978). Errors in classifying multivariate observations. 7heory of Probabilities and Applications, 23(4), $772-781$ (in Russian).

Raudys, S. (1972). On the amount of a priori information in designing the classification algorithm. Proc. Acad. of Sci. of USSR, Eng. Cybernetics, 14, 168-174 (in Russian).

Raudys, S. (1991). Methods for overcoming dimensionality problems in statistical pattern recognition. A review. Zavodskaya Laboratorya (Factory Lab., USSR Journal), Moscow: Nauka, 3, 45 \& 49-55 (in Russian).

Raudys, S. (1996). Lincar classifiers in perceptron design, ICPR13, Proc. 13th Imt. Conf, on Pattern Recognition (Vicnna. Austria, Aug.25-29) Vol. 4, Track D: Parallel and Conncctionist Systems, IEEE Computer Socicty Press, Los Alamitos, 1996, 763767.

Raudys, S. (1998). Evolulion and gencralization of a single neuronc. Part I. SLP as Seven statistical classificrs. Netral Networks (accepted).

Raudys, S. \& Jain, A.K. (1991). Small sample size effects in statistical pattern recognition: Recommendations for practitioners- IEEE Trans. on Pattern Analysis and Machine Intelligence, PAMI-13, 252-264.

Raudys, S. \& Pikelis, V.(1980). On dimensionality, sample size, classification error and complexity of the classification algorithm in pattern recognition. IEEE Trans. on Pattern Analysis and Machine Intelligence. PAMI-2 (3), 242-252. 